\title{
Management of severe asthma: summary of the European Respiratory Society/American Thoracic Society task force report
}

\section{Educational aims}

This article is mainly intended for trainees and specialists who are interested in the management of severe asthma.

- It aims to inform readers about the updated ERS/ATS recommendations for management of severe asthma, specifically on the topics of biologics, macrolides and long-acting muscarinic antagonists.

- It also provides guidance on utilisation of available biomarkers in selecting advanced therapies in severe asthma.

\section{Background}

Severe asthma is a heterogenous condition with many phenotypes identified in adults and children [1-4]. Until recently, asthma was considered predominantly a T-helper 2 cell (Th2) disease, characterised by allergic eosinophilic inflammation mediated by Th2 cytokines (interleukin (IL)-4, IL-5 and IL-13) and IgE. While the majority of early-onset and mild-moderate asthma exhibits Th2-biased inflammation, the inflammatory basis of severe asthma is more complex. Innate type- 2 lymphoid cells (ILC2), which secrete IL-5 and IL-13, play a role in nonallergic eosinophilic inflammation. The term "type 2" (T2) asthma has replaced "Th2 asthma" to include contributions from ILC2 and associated cytokines (figure 1). A substantial number of

@ERSpublications

This article summarises the 2019 @EuroRespSoc/@ATScommunity Severe Asthma Task Force recommendations on the use of anti-IL-5 strategy, anti-IL-4/13 antibody, tiotropium and macrolides in severe asthma, and the role of biomarkers in directing therapy https://bit.ly/3braK43 patients with severe asthma do not have markers of T2 inflammation (non-T2 or T2-low asthma). Currently available biological therapies only target T2 asthma. The immunological basis of non-T2 asthma is not fully understood and remains an area

The first European Respiratory Society (ERS)/ American Thoracic Society (ATS) task force report, published in 2014, reviewed the definition and provided guidance on evaluation and treatment of severe asthma in children and adults [5]. Severe asthma was defined as "asthma that requires treatment with high-dose inhaled corticosteroids plus a second controller and/or systemic corticosteroids to prevent it from becoming 'uncontrolled' or that remains 'uncontrolled' despite this therapy" [5]. The task force placed emphasis on excluding asthma of active research.
Cite as: Khurana S, Bush A, Holguin F. Management of severe asthma: summary of the European Respiratory Society/American Thoracic Society task force report. Breathe 2020; 16: 200058.

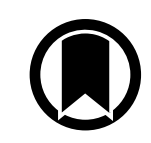

CrossMark

(C) ERS 2020 


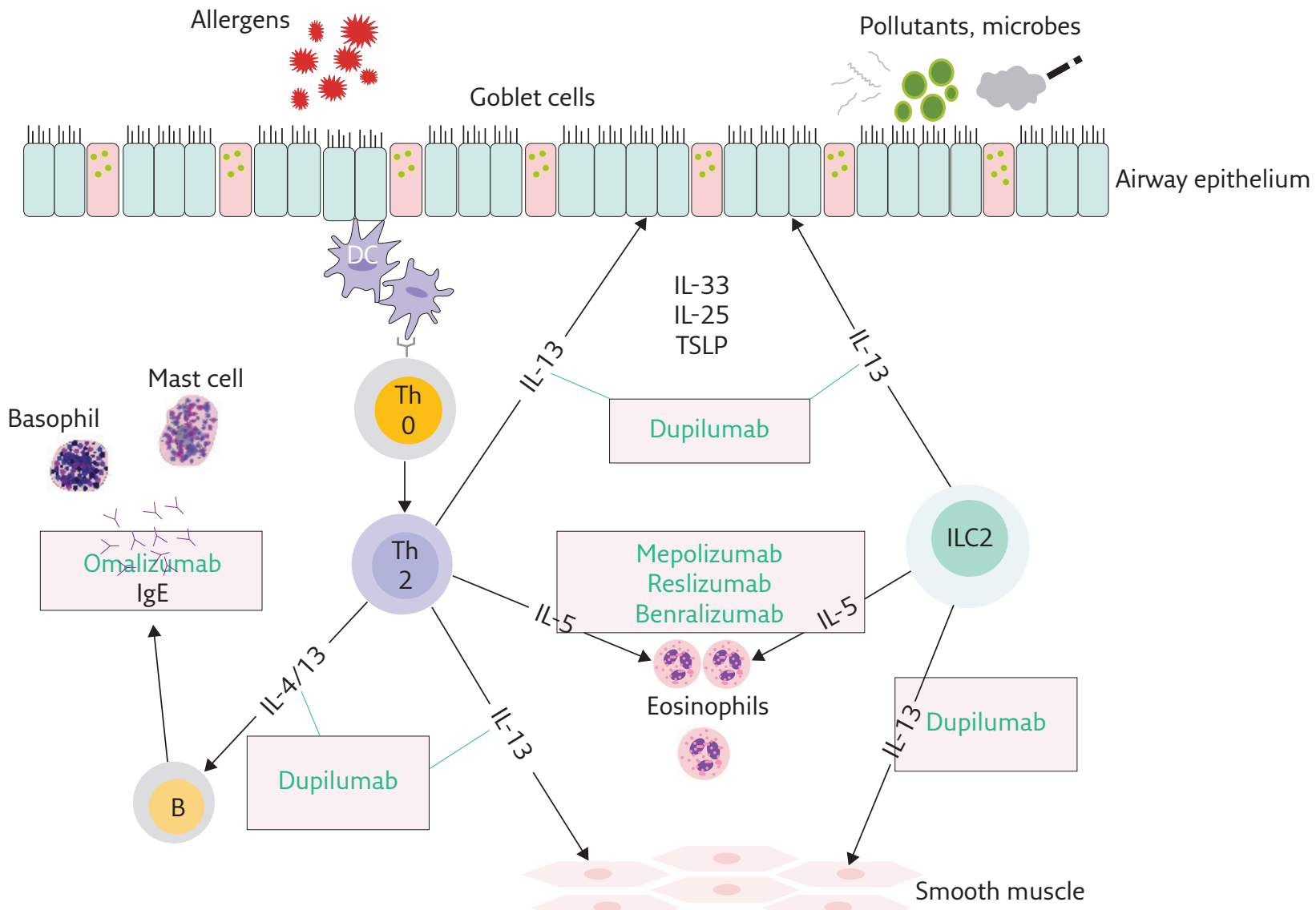

Figure 1 T2 inflammation in asthma and available biologics. DC: dendritic cell; ThO: naïve T-cell; Th2: T-helper 2 cell; B: B-cell; TSLP: thymic stromal lymphopoietin.

mimics and controlling comorbidities, particularly in difficult-to-treat asthma. The 2014 severe asthma guidelines addressed the use of computed tomography, certain biomarkers (sputum eosinophils and exhaled nitric oxide fraction $\left(F_{\mathrm{ENO}}\right)$ ), omalizumab, methotrexate, macrolides, antifungals and bronchial thermoplasty in severe asthma [5].

Four new biological therapies have become available for treatment of severe eosinophilic T2 asthma since the first task force report (table 1). Three biologics target the IL-5 pathway (mepolizumab, reslizumab and benralizumab). Dupilumab is an IL-4 receptor $\alpha(\mathrm{IL}-4 \mathrm{R} \alpha)$ antagonist,

Table 1 Currently available biologics for T2 asthma

\begin{tabular}{lll}
\hline Drug & Mechanism & Route \\
\hline Omalizumab & Anti-IgE & Subcutaneous \\
Mepolizumab & Anti-IL-5 & Subcutaneous \\
Reslizumab & Anti-IL-5 & Intravenous \\
Benralizumab & Anti-IL-5R $\alpha$ & Subcutaneous \\
Dupilumab & Anti-IL-4R $\alpha$ & Subcutaneous
\end{tabular}

R: receptor. and blocks the IL-4 and IL-13 pathway (figure 1). Results of randomised controlled trials (RCTs) have also been reported evaluating efficacy of long-acting muscarinic antagonists (LAMA) and macrolides in asthma.

\section{The Severe Asthma Task Force of the ERS/ATS}

The second joint ERS and ATS Severe Asthma Task Force was commissioned in 2017 to address new treatments for severe asthma that were introduced after the first task force report [6]. This task force included clinicians and researchers with expertise in severe asthma in adults and children, methodologists, and patient representatives. The task force specifically addressed six questions pertaining to management of severe asthma (table 2).

The rigorous GRADE (Grading of Recommendations Assessment, Development and Evaluation) approach was used for formulating questions, grading the evidence and deciding the strength of recommendations. Using this process, the task force developed evidence-based guidelines for these aspects of the management of severe asthma, full details of which were recently 
Table 2 Questions selected by the ERS/ATS Severe Asthma Task Force

1. Should a monoclonal anti-IL-5 antibody be used in adults and children ${ }^{\#}$ with severe asthma?

2. Should a measurement of a specific biomarker be used to guide initiation of treatment with a monoclonal anti-IL-5 or IL-5R $\alpha$ antibody in adults and children with severe asthma? (Chosen biomarkers being $F_{\mathrm{ENO}}$, peripheral or sputum eosinophils and serum periostin)

3. Should a measurement of a specific biomarker be used, in addition to total IgE level, to guide initiation of treatment with a monoclonal anti-lgE antibody in adults and children with severe asthma? (Chosen biomarkers being $F_{\mathrm{ENO}}$, peripheral or sputum eosinophils and serum periostin)

4. Should an inhaled LAMA be used in adults and children with severe asthma?

5. Should a macrolide (i.e. azithromycin, clarithromycin) be used in adults and children with severe asthma?

6. Should a monoclonal anti-IL-4R $\alpha$ be used in adults and children with severe asthma?

R: receptor. \#: for the purposes of this guideline, age $>5$ years. Reproduced and modified from [6].

published [6]. The aim of this article is to provide a concise review of the guidelines for use by trainees and secondary and tertiary care physicians.

\section{What questions did the task force consider?}

The ERS/ATS task force considered six questions focused on the use of new biologics in adults and children with severe asthma, the role of biomarkers in selecting such biologics, and the use of LAMA and macrolides in adults and children with severe asthma (table 2).

The treatments considered were:

- Antil-IL-5 therapies (mepolizumab, reslizumab and benralizumab)

- Anti-IL-4/13 antibody (dupilumab)

- LAMA

- Macrolides

The biomarkers considered were:

- Sputum eosinophils

- Blood eosinophils

- $F_{\mathrm{ENO}}$

- Serum periostin

\section{Should a monoclonal anti-IL5 or antil-IL-5 receptor $\alpha$-subunit antibody be used in adults and children with severe asthma?}

IL-5 plays a key role in eosinophil biology including recruitment, maturation and survival of eosinophils in severe asthma. Eosinophilic inflammation in asthma is associated with increased risk of exacerbations. Monoclonal antibodies that target the IL-5 pathway have been found to be effective in RCTs. There are currently three anti-IL-5 therapies approved by the US Food and Drug Administration (FDA) and the European Medicines Agency (EMA) for use in severe eosinophilic asthma.

- Mepolizumab, an anti-IL-5 monoclonal antibody, is administered at a fixed dose of $100 \mathrm{mg}$ subcutaneously every 4 weeks. In RCTs, mepolizumab therapy was associated with a $50 \%$ reduction in the rate of asthma exacerbations in patient with severe uncontrolled asthma and blood eosinophil count $\geq 300$ cells per $\mu \mathrm{L}$ in 12 months prior or $\geq 150$ cells per $\mu \mathrm{L}$ at screening $[7,8]$. Mepolizumab was also associated with $50 \%$ median reduction in the dose of maintenance oral corticosteroid (OCS) dose [9].

- Reslizumab is an anti-IL-5 monoclonal antibody administered as an intravenous infusion at a weight-based dose of $3 \mathrm{mg} \cdot \mathrm{kg}^{-1}$ every 4 weeks. In RCTs, reslizumab therapy was associated with a $54 \%$ reduction in the rate of any exacerbations and $33 \%$ reduction in the rate of exacerbations requiring emergency room visit or hospitalisation [10-13].

- Benralizumab binds to the $\alpha$-subunit of the IL-5 receptor (IL-5R $\alpha$ ). The dose is $30 \mathrm{mg}$ every 4 weeks for the initial three doses, followed by $30 \mathrm{mg}$ every 8 weeks, administered subcutaneously. In patients that met the ERS/ ATS criteria of severe asthma, benralizumab therapy resulted in a $55 \%$ reduction in the number of patients with exacerbations [14, 15]. Benralizumab was associated with $50 \%$ mean reduction in the dose of maintenance OCS dose in patients with OCS-dependent asthma [16].

When deliberating recommendations for use of anti-IL-5 therapy, the task force considered the evidence from 12 RCTs. The panel deemed the following outcomes as "critical": asthma exacerbations, asthma symptoms, asthma control, quality of life, systemic corticosteroid exposure and adverse events. Change in lung function was considered an "important" outcome. The ERS/ATS task force concluded that all anti-IL-5 therapies reduce exacerbations in patients with severe eosinophilic asthma. However, only mepolizumab and benralizumab have been systematically studied in patients with OCS-dependent asthma and were 
effective in reducing OCS maintenance dose. The effects of anti-IL-5 strategy on asthma control, quality of life and forced expiratory volume in $1 \mathrm{~s}\left(\mathrm{FEV}_{1}\right)$ were modest and did not meet the minimal clinically important difference (MCID) threshold. It is important to note that the studies included patients with varying degrees of asthma severity that did not consistently meet the ESR/ATS criteria for severe asthma.

\section{Task force recommendation}

The task force conditionally suggested the use of "an anti-IL-5 strategy as add-on therapy for adult patients with severe uncontrolled asthma with an eosinophilic phenotype and for those with severe corticosteroid-dependent asthma". The task force could not provide a recommendation in adolescents due to the limited number of participants in this age group across studies

\section{Should a monoclonal anti- IL-4R $\alpha$ be used in adults and children with severe asthma?}

Dupilumab is a monoclonal antibody that blocks signalling of two key T2 cytokines, IL-4 and IL-13, by binding to the $\alpha$-subunit of the IL- 4 receptor (IL-4R $\alpha$ ). The task force identified three RCTs of dupilumab in moderate-severe asthma, data from which were pooled for analysis [17-19]. One trial was in patients with OCS-dependent asthma [19]. Dupilumab add-on therapy was associated with a significant reduction in exacerbation frequency and OCS dependence. Statistically significant improvements were observed in $\mathrm{FEV}_{1}$, and Asthma Control Questionnaire (ACQ)-5 and Asthma Quality of Life Questionnaire (AQLQ) scores but did not reach the MCID. In one study that further stratified results by blood eosinophils and $F_{\mathrm{ENO}}$, the effect was greater in patients with blood eosinophils $\geq 150$ per $\mu \mathrm{L}$ or $F_{\text {ENO }} \geq 25$ ppb [18]. The task force concluded that "dupilumab add-on therapy substantially decreases exacerbations in moderate to severe uncontrolled asthma and is effective in reducing OCS dose in patients with severe OCS-dependent asthma".

\section{Task force recommendation}

The task force conditionally suggested use of dupilumab as add-on therapy for adult patients with severe eosinophilic asthma, and for patients with severe corticosteroiddependent asthma with or without eosinophilia. Again, the task force could not provide a recommendation in adolescents due to the limited number of treated patients in this age group.

\section{Should a LAMA be used in adults and children with severe asthma?}

The panel reviewed three RCTs in adults, one in adolescents and one in children (age 6-11 years) evaluating efficacy of tiotropium delivered via Respimat soft-mist inhaler [20-23]. The addition of tiotropium $5 \mu \mathrm{g}$ led to improvements in mean peak $\mathrm{FEV}_{1}$ and asthma control (measured by ACQ-7), as well as prevented worsening of asthma across all age groups. In adults, tiotropium $5 \mu \mathrm{g}$ did not result in significant differences in asthma quality of life measured by AQLQ, but did increase time to first exacerbation requiring OCS. The task force concluded that "LAMA treatment in children, adolescents and adults with severe asthma may improve $\mathrm{FEV}_{1}$ and may reduce loss of asthma control. In adults, treatment with tiotropium $5 \mu \mathrm{g}$ also improves asthma control and increases time to first exacerbation".

\section{Task force recommendation}

The task force recommended the addition of tiotropium in patients with severe asthma that is uncontrolled despite Global Initiative for Asthma (GINA) step 4/5 or National Asthma Education and Prevention Program (NAEPP) step 5 therapies. This was a strong recommendation with moderate quality of evidence.

\section{Should a macrolide be used in adults and children with severe asthma?}

The 2014 ERS/ATS guidelines, based on then available evidence, provided a conditional recommendation against the use of long-term macrolide antibiotics in adults or children with severe asthma [5]. The current task force evaluated six RCTs: five included adults and one included children 6 to $<18$ years of age [24-29]. Four studies evaluated azithromycin and two studies evaluated clarithromycin. Of note, the study subjects did not always meet ERS/ATS criteria for severity. In one study, azithromycin reduced the frequency of moderate and severe exacerbations, and increased the time to first exacerbation [25]. Macrolides did not, however, reduce the rate of severe exacerbations or improve lung function in children or adults. Similarly, macrolides did not improve asthma control (ACQ-7) or asthma quality of life (AQLQ) beyond the MCID. The task force concluded that "chronic macrolide therapy reduces the risk of having an asthma exacerbation. However, there is no conclusive evidence that treatment shows any effect in reducing severe 
exacerbations or hospitalisations". The task force further cautioned that "it is unknown whether this therapy will improve outcomes among those meeting ERS/ATS criteria for severe asthma". The risk of prolonged antibiotic exposure including emergence of resistant pathogens must be weighed against potential benefit when considering such therapy. It is also important to note that safety and efficacy of macrolides in asthma beyond 1 year have not been studied.

\section{Task force recommendation}

The task force suggested a trial of macrolide treatment to reduce asthma exacerbations in adult asthma subjects on GINA/NAEPP step 5 therapy that remain persistently symptomatic or uncontrolled. The task force suggested against the use of chronic macrolide treatment in children and adolescents with severe uncontrolled asthma. These were conditional recommendations with low quality of evidence.

\section{Biomarkers in severe asthma}

The task force considered four biomarkers: sputum eosinophils, blood eosinophils, $F_{\mathrm{ENO}}$ and serum periostin (figure 2). Sputum eosinophils have traditionally been used to identify patients with eosinophilic asthma with a cut-off of $\geq 3 \%$ generally accepted as significant. However, sputum induction and processing for eosinophils can be time consuming and not always successful. Sputum analysis, therefore, remains largely a research tool available at select asthma centres. Blood eosinophils have been used successfully to select patients with severe eosinophilic asthma in recent clinical trials of anti-IL5 and anti-IL-4/13 biologics. Low cost and ease of measurement make them an attractive surrogate biomarker of eosinophilic inflammation. A single low blood eosinophil value, however, should be interpreted with caution as blood eosinophils can be highly variable and affected by OCS or high-dose inhaled corticosteroid therapy. $F_{\mathrm{ENO}}$ is a noninvasive biomarker of airway inflammation associated with IL-13, allergic and eosinophilic inflammation. It can be easily measured at the point of care in adults and children, and is currently available for clinical use. Periostin is an extracellular matrix protein involved in homeostatic and disease processes. In asthma, it is associated with increased IL-4/IL-13 activity and eosinophilic airway inflammation. Periostin levels are influenced by age, skeletal growth and puberty, and there is evidence that it is not a suitable biomarker in children and adolescents. Serum periostin was used as a surrogate biomarker of Th2 inflammation in clinical trials evaluating an anti-IL-13 biologic [30]. It is not currently available for clinical use.

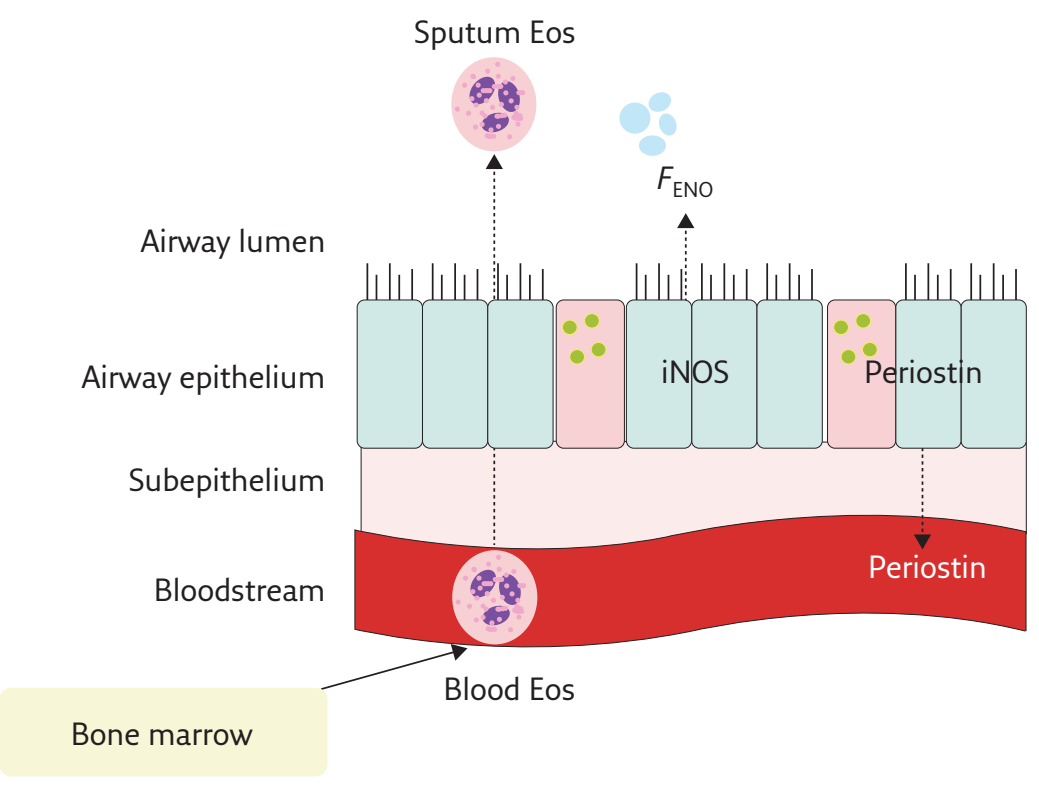

Figure 2 Biomarkers in T2 asthma. Eos: Eosinophil; iNOS: inducible nitric oxide synthase.

\section{Should a specific biomarker be used to initiate treatment with an anti-IL-5 therapy in severe asthma?}

The task force identified 12 RCTs of anti-IL-5 therapies in children and adults that evaluated treatment response based on eosinophil cut-offs [8, 10-16, 31-33]. Blood eosinophil count was the most commonly used biomarker although threshold varied across anti-IL-5 strategies. Only one study evaluated sputum eosinophils. The task force concluded that "although the data suggest that subjects with higher levels of blood eosinophil counts benefit more from anti-IL-5 strategies, the evidence reviewed did not show that a specific level of blood eosinophils ( $\geq 150$ per $\mu \mathrm{L}$ for mepolizumab, $\geq 300$ per $\mu \mathrm{L}$ for benralizumab and $\geq 400$ per $\mu \mathrm{L}$ for reslizumab) is an absolute response threshold". It is important to note that eosinophil counts can vary considerably over time and repeated measurements may be necessary. Treatment effect, particularly from use of OCS or high-dose inhaled corticosteroids, must also be considered when evaluating patients' eosinophil status.

\section{Task force recommendation}

The task force conditionally suggested that a blood eosinophil threshold of $\geq 150$ per $\mu \mathrm{L}$ can be used to guide initiation of anti-IL-5 therapy in adults with severe asthma and a history of asthma exacerbations. 


\section{Should measurement of a specific biomarker, in addition to total IgE level, be used to guide initiation of treatment with anti-IgE antibody in severe asthma?}

Omalizumab is a monoclonal anti-lgE antibody approved by the FDA (in 2003) and EMA (in 2005) as add-on therapy for management of severe allergic asthma. It is administered subcutaneously every 2-4 weeks at a dose determined by patient's weight and total serum IgE level. It has been extensively evaluated in RCTs, and found to be efficacious in decreasing exacerbation frequency and improving asthma symptom scores as well as quality of life [34]. The first ERS/ATS task force report recommended use of omalizumab as add-on therapy in adults and children with severe allergic asthma [5]. The current task force evaluated utility of biomarkers other than serum IgE in guiding anti-IgE treatment. The group identified three RCTs that performed subgroup analyses based on blood eosinophils, $F_{\mathrm{ENO}}$ and serum periostin [35-37]. Higher blood eosinophils were associated with a greater improvement in lung function and exacerbations. Similarly, higher $F_{\text {ENO }}$ was associated with improvement in asthma quality of life and exacerbations. Higher serum periostin levels, however, did not correlate with better outcomes. The task force concluded that "blood eosinophil counts and $F_{\mathrm{ENO}}$ levels may be useful in choosing patients most likely to achieve a more positive effect on exacerbations and lung function when treated with omalizumab compared with placebo".

\section{Task force recommendation}

The task force conditionally suggested utilising a blood eosinophil threshold of $\geq 260$ per $\mu \mathrm{L}$ and $F_{\mathrm{ENO}} \geq 19.5 \mathrm{ppb}$ to identify patients more likely to benefit from omalizumab therapy. These recommendations were made with an important caveat that these cut-offs were determined largely by one study and have not been prospectively tested. Patients with biomarker levels below the proposed thresholds may still benefit from anti-lgE therapy.

Table 3 Summary of ERS/ATS severe asthma task force recommendations

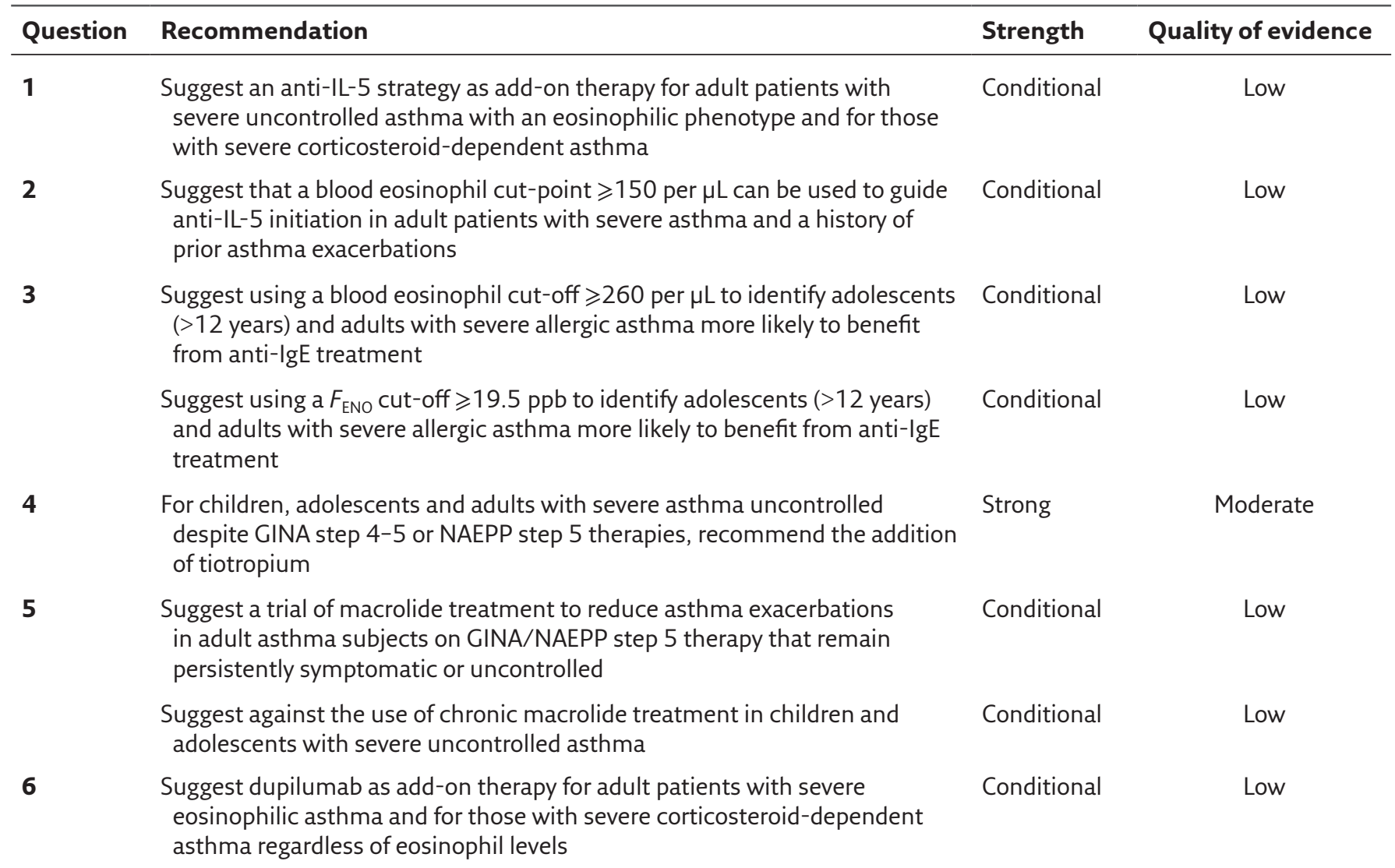

Reproduced and modified from [6]. 


\section{Conclusions and future perspectives}

The second joint ERS/ATS severe asthma task force systematically addressed six questions relating to the use of biomarkers and advanced treatments in severe asthma. A summary of recommendations is provided in table 3 . A conditional recommendation was made for the use of anti-IL-5 and anti-IL-4/13 therapies in patients with severe eosinophilic asthma, and for anti-IL-4/13 antibody in patients with OCS-dependent asthma regardless of eosinophil count. Recommendations were also made for utilisation of specific biomarker thresholds to identify patients most likely to respond to anti-IL-5 or anti-IgE therapy. Inhaled tiotropium was recommended for children and adults with severe asthma that remains uncontrolled despite high-intensity therapy. A trial of macrolides was conditionally suggested in adult patients with uncontrolled asthma on step 5 therapy while simultaneously weighing the potential benefit of such therapy against the risk of emergence of antimicrobial resistance.

While deliberating each question, the task force considered the benefit of the intervention versus the burden (adverse effects and cost), quality of evidence, feasibility and acceptability. The recommendations were based on the best evidence at this time and should be reconsidered when new evidence becomes available. Understanding the context and caveats surrounding these recommendations is critical as clinicians prepare to incorporate them into practice. Before applying these new and often expensive interventions, every effort must be made to use standard therapies to maximum benefit, ensure that patients are adherent with prescribed treatments and comorbidities have been optimally addressed. Many of the advanced diagnostics and treatments may only be available through specialised centres. It is important that all clinicians who care for patients with asthma can identify the patients most likely to benefit from referral to specialist care.

With an ever-increasing choice of biologics for treatment of T2 asthma, pragmatic clinical trials are needed to guide selection of the most effective therapy with improved precision. RCTs are also needed to evaluate the ability of individual and composite biomarkers to predict response to treatment for clinically important outcomes. Furthermore, we need biomarkers that can aid clinical decision making in terms of continuing versus stopping of specific therapies. While we have made significant progress in management of patients with T2 asthma, there is an urgency for future research to focus on treatments for non-T2 severe asthma as this remains an area of great unmet need.

\section{Key points}

- Severe asthma is defined as "asthma that requires treatment with high-dose inhaled corticosteroids plus a second controller and/or systemic corticosteroids to prevent it from becoming 'uncontrolled' or that remains 'uncontrolled' despite this therapy".

- In 2019, the European Respiratory Society (ERS)/American Thoracic Society (ATS) Severe Asthma Task Force published recommendations on the management of severe asthma, specifically on the use of anti-interleukin (IL)-5 strategy, anti-IL-4/13 antibody, long-acting muscarinic antagonists and macrolides, and the role of biomarkers in directing therapy.

1. The task force suggested the use of an anti-IL-5 strategy as add-on therapy in adult patients who have severe uncontrolled eosinophilic asthma and for those with severe corticosteroiddependent asthma.

2. The task force suggested the use of dupilumab as add-on treatment for adult patients with severe asthma of eosinophilic phenotype and for those with severe corticosteroid-dependent asthma irrespective of eosinophil status.

3. The task force recommended tiotropium as add-on treatment in children, adolescents and adults with severe asthma that remains uncontrolled despite Global Initiative for Asthma (GINA) step 4/5 or National Asthma Education and Prevention Program (NAEPP) step 5 therapy.

4. The task force suggested a trial of a macrolide in adult patients with severe asthma that remains uncontrolled on GINA/NAEPP step 5 therapy. The potential benefit from macrolide therapy 
must be weighed against the significant individual and public health risk of emergence of antimicrobial resistance. The task force suggested against the use of chronic macrolide therapy in children and adolescents with severe uncontrolled asthma.

- The task force also made recommendations on the utility of biomarkers in selecting advanced therapies in severe asthma.

1. The task force suggested that a blood eosinophil cut-off of $\geq 150$ per $\mu \mathrm{L}$ can be used to guide initiation of anti-IL-5 therapy in adult patients with severe asthma and a history of frequent exacerbations.

2. The task force suggested a blood eosinophil cut-off $\geq 260$ per $\mu \mathrm{L}$ and an exhaled nitric oxide fraction cut-off $\geq 19.5 \mathrm{ppb}$ to identify adolescents and adults with severe allergic asthma more likely to benefit from anti-IgE treatment.

\section{Affiliations}

Sandhya Khurana ${ }^{1}$, Andrew Bush ${ }^{2}$ and Fernando Holguin ${ }^{3}$ on behalf of the European Respiratory Society/American Thoracic Society Severe Asthma Task Force

1 University of Rochester School of Medicine and Dentistry, Pulmonary and Critical Care Medicine, Rochester, NY, USA. ${ }^{2}$ Dept of Paediatrics, National Heart \& Lung Institute, Imperial College London, London, UK. ${ }^{3}$ University of Colorado, Pulmonary Sciences and Critical Care Medicine, Denver, CO, USA.

Conflict of interest

S. Khurana reports grants from GSK and Sanofi outside the submitted work. A. Bush has nothing to disclose. F. Holguin has nothing to disclose.

\section{References}

1. Moore WC, Meyers DA, Wenzel SE, et al. Identification of asthma phenotypes using cluster analysis in the Severe Asthma Research Program. Am J Respir Crit Care Med 2010; 181: 315-323.

2. Haldar P, Pavord ID, Shaw DE, et al. Cluster analysis and clinical asthma phenotypes. Am J Respir Crit Care Med 2008; 178 : 218-224.

3. Shaw DE, Sousa AR, Fowler SJ, et al. Clinical and inflammatory characteristics of the European U-BIOPRED adult severe asthma cohort. Eur RespirJ 2015; 46: 1308-1321.

4. Fitzpatrick AM, Teague WG, Meyers DA, et al. Heterogeneity of severe asthma in childhood: confirmation by cluster analysis of children in the National Institutes of Health/National Heart, Lung, and Blood Institute Severe Asthma Research Program. J Allergy Clin Immunol 2011; 127: 382-389.

5. Chung KF, Wenzel SE, Brozek JL, et al. International ERS/ATS guidelines on definition, evaluation and treatment of severe asthma. Eur Respir J 2014; 43: 343-373.

6. Holguin F, Cardet JC, Chung KF, et al. Management of severe asthma: a European Respiratory Society/American Thoracic Society guideline. Eur Respir J 2020; 55: 1900588.

7. Ortega HG, Liu MC, Pavord ID, et al. Mepolizumab treatment in patients with severe eosinophilic asthma. N Engl J Med 2014; 371: 1198-1207

8. Chupp GL, Bradford ES, Albers FC, et al. Efficacy of mepolizumab add-on therapy on health-related quality of life and markers of asthma control in severe eosinophilic asthma (MUSCA): a randomised, double-blind, placebo-controlled, parallel-group, multicentre, phase $3 \mathrm{~b}$ trial. Lancet Respir Med 2017; 5: 390-400.

9. Bel EH, Wenzel SE, Thompson PJ, et al. Oral glucocorticoidsparing effect of mepolizumab in eosinophilic asthma. N Engl J Med 2014; 371: 1189-1197.
10. Bjermer L, Lemiere C, Maspero J, et al. Reslizumab for inadequately controlled asthma with elevated blood eosinophil levels: a randomized phase 3 study. Chest 2016; 150: 789-798.

11. Castro M, Mathur S, Hargreave F, et al. Reslizumab for poorly controlled, eosinophilic asthma: a randomized, placebocontrolled study. Am J Respir Crit Care Med 2011; 184: 1125-1132.

12. Castro M, Zangrilli J, Wechsler ME, et al. Reslizumab for inadequately controlled asthma with elevated blood eosinophil counts: results from two multicentre, parallel, double-blind, randomised, placebo-controlled, phase 3 trials. Lancet Respir Med 2015; 3: 355-366.

13. Corren J, Weinstein S, Janka L, et al. Phase 3 Study of reslizumab in patients with poorly controlled asthma: effects across a broad range of eosinophil counts. Chest 2016; 150: 799-810.

14. Bleecker ER, FitzGerald JM, Chanez P, et al. Efficacy and safety of benralizumab for patients with severe asthma uncontrolled with high-dosage inhaled corticosteroids and long-acting $\beta_{2}$-agonists (SIROCCO): a randomised, multicentre, placebo-controlled phase 3 trial. Lancet 2016; 388: 2115-2127

15. FitzGerald JM, Bleecker ER, Nair P, et al. Benralizumab, an anti-interleukin- 5 receptor $\alpha$ monoclonal antibody, as add-on treatment for patients with severe, uncontrolled, eosinophilic asthma (CALIMA): a randomised, double-blind, placebocontrolled phase 3 trial. Lancet 2016; 388: 2128-2141.

16. Nair P, Wenzel S, Rabe KF, et al. Oral glucocorticoid-sparing effect of benralizumab in severe asthma. N EnglJ Med 2017; 376: 2448-2458

17. Wenzel S, Castro M, Corren J, et al. Dupilumab efficacy and safety in adults with uncontrolled persistent asthma despite 
use of medium-to-high-dose inhaled corticosteroids plus a long-acting $\beta 2$ agonist: a randomised double-blind placebocontrolled pivotal phase $2 \mathrm{~b}$ dose-ranging trial. Lancet 2016; 388: 31-44

18. Castro M, Corren J, Pavord ID, et al. Dupilumab efficacy and safety in moderate-to-severe uncontrolled asthma. N EnglJ Med 2018; 378: 2486-2496.

19. Rabe KF, Nair P, Brusselle G, et al. Efficacy and safety of dupilumab in glucocorticoid-dependent severe asthma. $N$ EnglJ Med 2018; 378: 2475-2485.

20. Kerstjens HA, Disse B, Schröder-Babo W, et al. Tiotropium improves lung function in patients with severe uncontrolled asthma: a randomized controlled trial. J Allergy Clin Immunol 2011; 128: 308-314.

21. Kerstjens HA, Engel M, Dahl R, et al. Tiotropium in asthma poorly controlled with standard combination therapy. NEngl J Med 2012; 367: 1198-1207.

22. Hamelmann E, Bernstein JA, Vandewalker M, et al. A randomised controlled trial of tiotropium in adolescents with severe symptomatic asthma. Eur Respir J 2017; 49: 1601100.

23. Szefler SJ, Murphy K, Harper T, et al. A phase III randomized controlled trial of tiotropium add-on therapy in children with severe symptomatic asthma. J Allergy Clin Immunol 2017; 140: 1277-1287

24. Brusselle GG, Vanderstichele C, Jordens P, et al. Azithromycin for prevention of exacerbations in severe asthma (AZISAST): a multicentre randomised double-blind placebo-controlled trial. Thorax 2013; 68: 322-329.

25. Gibson PG, Yang IA, Upham JW, et al. Effect of azithromycin on asthma exacerbations and quality of life in adults with persistent uncontrolled asthma (AMAZES): a randomised, double-blind, placebo-controlled trial. Lancet 2017; 390: 659-668.

26. Strunk RC, Bacharier LB, Phillips BR, et al. Azithromycin or montelukast as inhaled corticosteroid-sparing agents in moderate-to-severe childhood asthma study. J Allergy Clin Immunol 2008; 122: 1138-1144.
27. Hahn DL, Grasmick M, Hetzel S, et al. Azithromycin for bronchial asthma in adults: an effectiveness trial. J Am Board Fam Med 2012; 25: 442-459.

28. Sutherland ER, King TS, Icitovic N, et al. A trial of clarithromycin for the treatment of suboptimally controlled asthma. J Allergy Clin Immunol 2010; 126: 747-753.

29. Simpson JL, Powell H, Boyle MJ, et al. Clarithromycin targets neutrophilic airway inflammation in refractory asthma. Am J Respir Crit Care Med 2008; 177: 148-155.

30. Corren J, Lemanske RF, Hanania NA, et al. Lebrikizumab treatment in adults with asthma. N Engl J Med 2011; 365: 1088-1098.

31. Castro M, Wenzel SE, Bleecker ER, et al. Benralizumab, an antiinterleukin 5 receptor $\alpha$ monoclonal antibody, versus placebo for uncontrolled eosinophilic asthma: a phase $2 \mathrm{~b}$ randomised dose-ranging study. Lancet Respir Med 2014; 2: 879-890.

32. Park HS, Kim MK, Imai N, et al. A phase 2a study of benralizumab for patients with eosinophilic asthma in south korea and japan. Int Arch Allergy Immunol 2016; 169: 135-145.

33. Ortega HG, Yancey SW, Mayer B, et al. Severe eosinophilic asthma treated with mepolizumab stratified by baseline eosinophil thresholds: a secondary analysis of the DREAM and MENSA studies. Lancet Respir Med 2016; 4: 549-556.

34. Normansell R, Walker S, Milan SJ, et al. Omalizumab for asthma in adults and children. Cochrane Database Syst Rev 2014; CD003559.

35. Ledford D, Busse W, Trzaskoma B, et al. A randomized multicenter study evaluating Xolair persistence of response after long-term therapy. J Allergy Clin Immunol 2017; 140: 162-169.

36. Busse W, Spector S, Rosén K, et al. High eosinophil count: a potential biomarker for assessing successful omalizumab treatment effects. J Allergy Clin Immunol 2013; 132: 485-486.

37. Hanania NA, Wenzel S, Rosén K, et al. Exploring the effects of omalizumab in allergic asthma: an analysis of biomarkers in the EXTRA study. Am J Respir Crit Care Med 2013; 187: 804-811. 